\section{Sharing pharma compounds with academia: experiences with providing vitamin D receptor ligands}

\author{
Arndt A. Schmitz, Sandra Hackethal, Anke Schulz, Ekkehard May, \\ Andreas Steinmeyer, Ulrich Zügel and Khusru Asadullah
}

Open innovation, a strategy popularized by Henry Chesbrough more than a decade ago (Nature Rev. Drug Discov. 12, 338-339 $(2013))^{1}$, is an important and promising trend in drug research and development $(\mathrm{R} \& \mathrm{D})^{2,3}$. One example is donating compounds from pharmaceutical research groups to academia. Over the last 20 years, we have provided compounds from discovery projects to academic scientists for research purposes, with vitamin D receptor (VDR) ligands being the cluster most frequently donated.

The VDR is a nuclear receptor and a clinically validated drug target ${ }^{4}$. It acts as a transcription factor, in particular on mineral metabolism pathways. The naturally occurring ligand of the VDR, vitamin $\mathrm{D}$, is a fat-soluble prohormone that is synthesized in the skin in response to sunlight. Its active form, calcitriol, and synthetic analogues (such as calcipotriol) are approved treatments for numerous diseases, including psoriasis, a common autoimmune disorder ${ }^{4}$.

Since 1989, the former Schering AG (now part of Bayer AG) has synthesized, characterized and published research on novel ligands of the VDR in peer-reviewed journals ${ }^{5}$. Among these ligands is the unique VDR antagonist, ZK159222 (REF. 6). This and other compounds in its class have proved useful as tools - for example, for analysing molecular mechanisms in immune reactions ${ }^{7}$. Internal drug discovery efforts to target the VDR were discontinued owing to strategic considerations, but the ligands have been donated to many academic groups for research purposes. Here, we present a retrospective analysis of this programme, including the results of a survey of 63 recipients.

\section{Internal analysis}

We analysed the current in-house database (Electronic Substance Delivery Workflow; Lotus Notes, IBM), in which all deliveries of substances from any internal research programme to external partners are documented. Approximately $5 \%$ of all deliveries were compound donations, with our proprietary VDR ligands being the most frequently donated. Information from other, legacy databases, as well as from the paper and electronic archives of the logistics department and from the scientists involved was also included. We also searched PubMed and Google Scholar for papers that used the donated VDR ligands.

Since 1994, 218 actual VDR ligand donations were made to 132 individual scientists worldwide (FIG. 1a,b). Approximately onethird of the ligand donations resulted in a peer-reviewed, original publication $(n=65$ papers, FIG. 1a). The mean time between donation of the compound and publication was 22 months. Overall, these papers have been cited over 5,100 times; a single paper that was published in Science has been cited over 1,800 times ${ }^{7}$. Even excluding this paper, each publication using our VDR ligands has been cited an average of 51 times (data not shown).

A more detailed analysis was performed for the period 2003-2013. During this time, a total of 121 requests were received (FIG. 1c). Briefly, to request compounds, the academic group completes a one-page non-confidential research project plan that is then reviewed by Bayer scientists. In addition, the academic institution has to review a sample transfer agreement (STA). The compound is dispatched if there are no reasonable objections against the research plan and if the STA is signed and returned.

The overall success rate for requests was high (71\%, or 86 of 121 requests), although there were regional differences. Requesters from the United States had a lower chance of receiving the compound than did requesters from other countries (FIG. 1d).

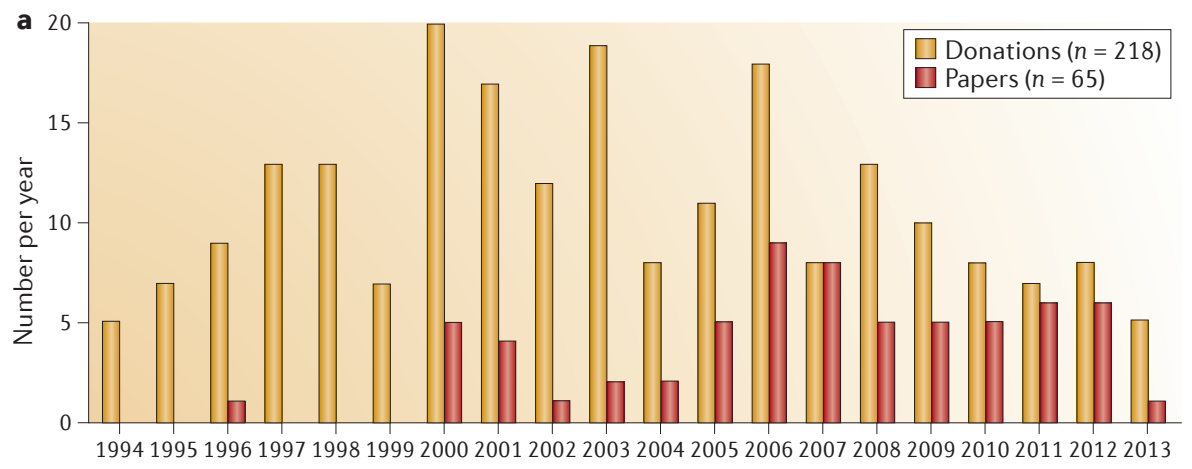

b

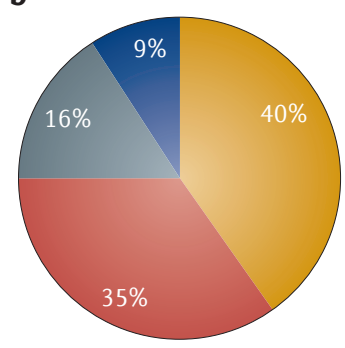

$\square$ Germany

$\square$ Europe (without Germany) $\square$ USA

Rest of world
C
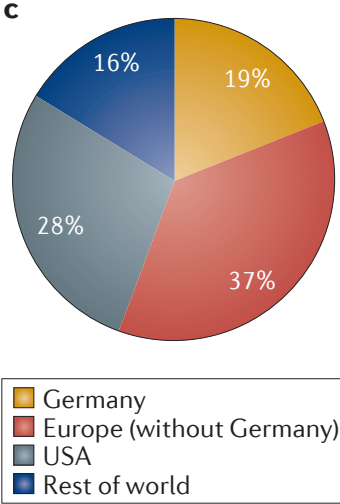

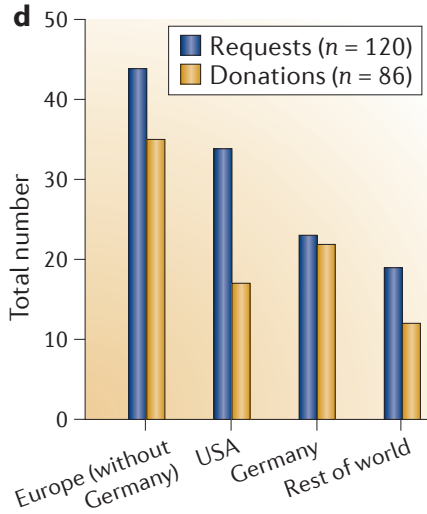

Figure 1 | Statistics for vitamin D receptor compound-donation programme. a|Annual VDR compound donations and resulting publications since 1994. b | Geographic distribution of vitamin D receptor (VDR) compound donations since 1994 ( $n=218$ donations). c | Geographic distribution of VDR compound requests since 2003 ( $n=120$ requests; details for one request not available). d) Requests versus actual donations of VDR compounds since 2003, analysed according to geographic location of the requesting scientist. 
Based on archived e-mail communications, the review and execution of the STA was the main cause of delay. Quite frequently, US institutions had either questions or concerns about the STA, or the US investigator never returned the STA template to Bayer.

A first request from an academic scientist for a compound from the VDR ligand cluster took an average of 99 days to fulfil (data on 19 requests available). This time was mostly spent on processing the STA. A second request from the same scientist took an average of just 57 days to fulfil ( $n=11$ requests). Even when successful, requests from the United States took substantially longer, with an average request time of 152 days ( $n=4$ requests) compared with 85 days for non-US institutions $(n=15$ requests).

\section{External survey}

To get an impression of the external scientists' perspective, we performed a brief survey of the recipients' profiles and experiences with obtaining compounds from, and communicating with, Bayer and other pharmaceutical companies.

Sixty-three scientists who had received a VDR compound between 2003 and 2013, and whose current e-mail addresses were available, were asked to complete the survey (via fax, PDF or anonymous web-link). Forty-six responded, yielding a return rate of $73 \%$. The majority of respondents used the anonymous web-link ( $n=38$ respondents; $83 \%$ ), reducing the risk of bias in their replies. The respondents were mostly principal investigators (69\%), frequently working in immunology (36\%), and requested special reagents from pharmaceutical companies about once a year $(65 \%)$.

The compound-donation programme was well appreciated; two-thirds of the recipients answered the question "How important was our compound donation to your research?" with "crucial" $(20 \%)$ or "significant" (43\%) (FIG. 2a). Whereas in general the quality of the communication between industry and academia received mixed ratings, with the answers split roughly equally into "poor" and "fair" versus "good" and "excellent" (data not shown), the compound-donation programme was rated positively overall (FIG. 2b); the effort to obtain the compound was considered to be no greater than "moderate" by $98 \%$ of the respondents (FIC. 2c) and the time span from request to delivery was usually deemed to be "as expected" or even "shorter than expected" (FIG. 2d).
Remarkably, the interactions within this compound-donation programme had a positive influence on the recipient's opinion of both pharmaceutical research in general and of Bayer in particular. This was revealed by asking "What change did our interaction bring to your image of pharmaceutical research in general?" and "[...] of Bayer Pharmaceutical in particular?” (FIG. 2e). There was a statistically significant positive relationship between the perceived quality of the interaction and the change of Bayer's image $(p=0.0007$, likelihood ratio chi-square test). The two aspects of the interaction that had the most significant influence on the perceived quality of the process were "willingness to support the recipients' research" ( $p=0.004$, likelihood ratio chi-square test) and "ease of contacting the correct person" ( $p=0.02$, likelihood ratio chi-square test); by contrast, the "STA" and "speed of reply" had no significant influence on the perceived quality.
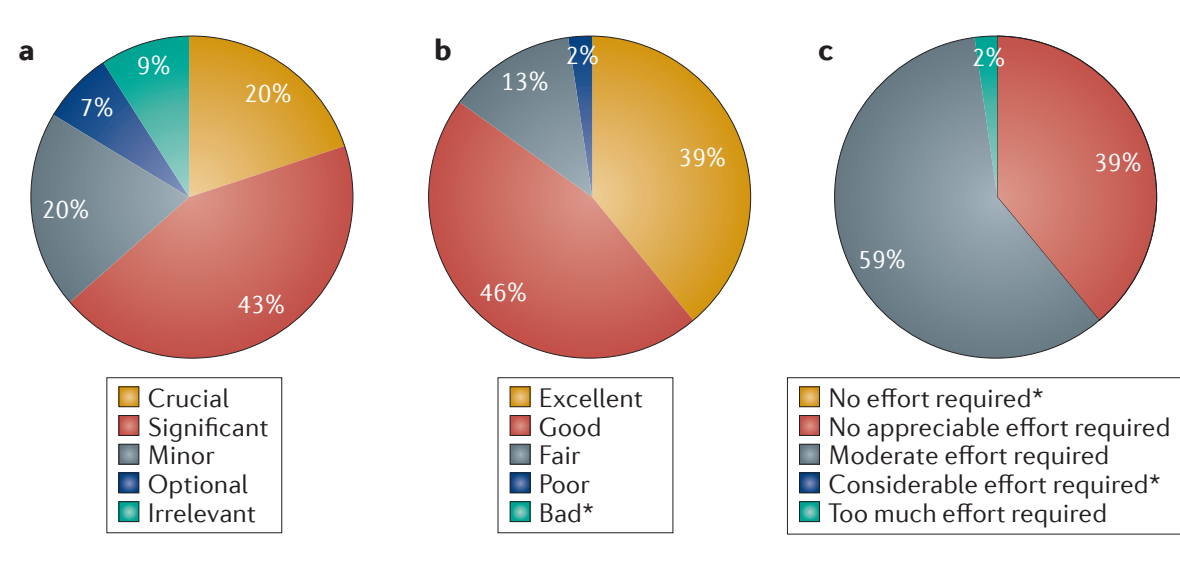

The vast majority (up to 95\%) of pharmaceutical R\&D projects fail at some stage of the process, making drug discovery a risky and expensive venture. Internal compound libraries therefore contain many substances - often with well-characterized pharmacology - that are no longer the focus of R\&D efforts. However, these compounds could serve a different purpose and be considered a potential 'gold mine' for basic research. Indeed, compound recipients were quite positive about the programme and frequently emphasized the value of the compounds provided for their research.

Our analysis also showed that about onethird of the VDR compound donations led (after an average lag phase of almost 2 years) to publications in peer-reviewed scientific journals, including in top-tier journals, and subsequently to many citations, further indicating the value of the findings for the scientific community. As a comparison,
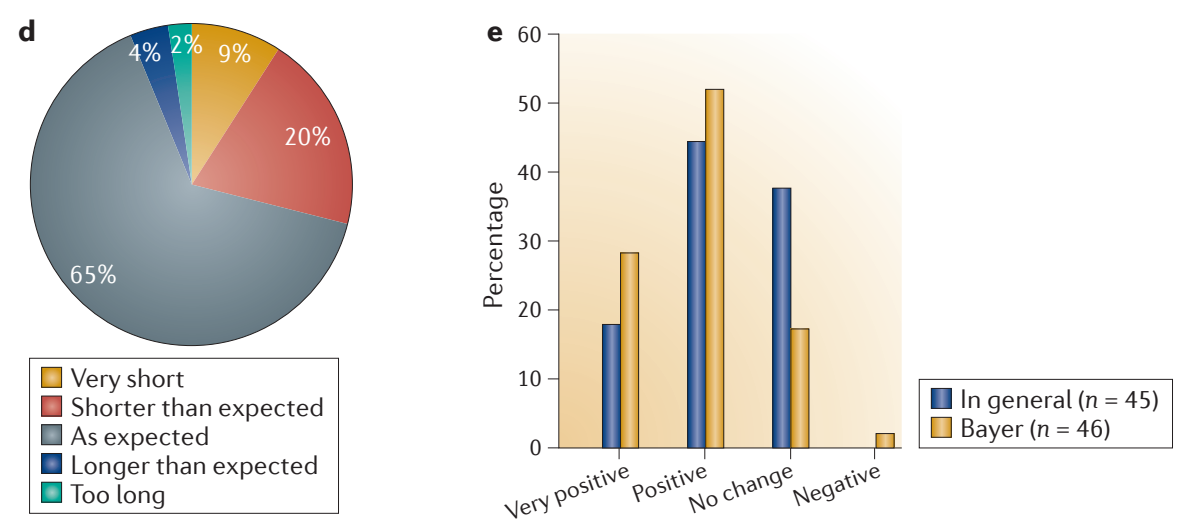

Figure 2 | Survey of value of vitamin $\mathrm{D}$ receptor compound donation programme for compound recipients. Answers to questions from the survey. a| "How important was our compound donation to your research?" ( $n=44$ responses). b| "What was your overall impression of the process of obtaining the requested compound from Bayer?" ( $n=46$ responses). c| "How much effort was required to obtain the compound from Bayer?" ( $n=46$ responses). $\mathbf{d}$ | "Did you deem the time span from request to delivery..." ( $n=46$ responses). e| "What change did our interaction bring to your image of pharmaceutical research in general?" ( $n=45$ responses) and "What change did our interaction bring to your image of Bayer Pharmaceutical in particular?" ( $n=46$ responses). *No responses in this category. 
the Structural Genomics Consortium (SGC) was recently repositioned to identify novel preclinical ligands and foster their availability ${ }^{8}$. They reported the publication impact — assessed using the h-index of such chemical probes. Interestingly, the nine probes the SGC reported had an average h-index of 29 , which is comparable to the h-index of 34 that we calculated for the VDR ligands.

One major obstacle for open innovation was identified through our analysis. Although Bayer is open to providing compounds to requesting academic partners, the execution of the STA was frequently a hurdle, particularly for US institutions. In order to alleviate this potential difficulty, Bayer has recently modified the STA. However, it is too early to determine whether this change (which was implemented in 2012) is increasing the success rate of current requests. Discussion of legal aspects was also the biggest obstacle for a joint academic-industrial clinical drug repurposing scheme, the Clinical and Translational Science Award programme?.

Compounds that have been deprioritized by the pharmaceutical industry for clinical development may start a second career as research tools for academia, leading to an improved understanding of molecular biology and mechanisms of diseases, and potentially contributing to novel therapeutic approaches. Such research activities may also help the repositioning the compounds for other, previously unconsidered indications ${ }^{3}$. Thus, it is in the best interest of pharmaceutical companies to enable such activities. Indeed, our data suggest that a compound-donation programme can be a valuable resource that is readily accepted and of great interest to many scientific communities.

Overall, we had good experiences in fostering open innovation, not just by providing compounds but also, for example, by granting funds for the validation of drug targets and biomarkers ${ }^{10}$. What seems to be a key success factor, however, is the close interaction with the academic groups. This should include frequent communication and, ideally, joined activities instead of just providing a compound or a grant ${ }^{2}$. We are currently considering broadening and further simplifying access to tool compounds for academic research groups.

Arndt A. Schmitz, Sandra Hackethal, Anke Schulz, Ekkehard May, Andreas Steinmeyer, Ulrich Zügel and Khusru Asadullah are at Global Drug Discovery, Bayer HealthCare Pharmaceuticals, Muellerstrasse 178, 13342 Berlin, Germany. A.A.S. and S.H. contributed equally to this work.
Correspondence to A.A.S e-mail:arndt.schmitz@bayer.com

doi: 10.1038/nrd4008-c1

1. Mullard, A. An audience with... Henry Chesbrough Nature Rev. Drug Discov. 12, 338-339 (2013).

2. Lessl, M. et al. Crowd sourcing in drug discovery. Nature Rev. Drug Discov. 10, 241-242 (2011).

3. Collins, F. S. Mining for therapeutic gold. Nature Rev. Drug Discov. 10, 397 (2011).

4. Feldman, D., Pike, J. W. \& Adams, J. S. (eds) Vitamin D (Academic Press, 2011).

5. Steinmeyer, A. et al. Synthesis and biological activities of 8(14) $\alpha$-homocalcitriol. Steroids 57, 447-452 (1992).

6. Herdick, M. et al. Antagonistic action of a 25-carboxylic ester analogue of $1 \alpha, 25$-dihydroxyvitamin D3 is mediated by a lack of ligand-induced vitamin $D$ receptor interaction with coactivators. J. Biol. Chem. 275, 16506-16512 (2000).

7. Liu, P. T. et al. Toll-like receptor triggering of a vitamin D-mediated human antimicrobial response. Science 311, 1770-1773 (2006).

8. Edwards, A. M. et al. Open access chemical and clinical probes to support drug discovery. Nature Chem. Biol. 5, 436-440 (2009).

9. Marusina, K. et al. The CTSA Pharmaceutical Assets Portal - a public-private partnership model for drug repositioning. Drug Discov. Today 8, 77-83 (2011).

10. Dorsch, H. et al. Grants4Targets: an open innovation initiative to foster drug discovery collaborations between academia and the pharmaceutical industry. Nature Rev. Drug Discov. 14, 74-76 (2015).

Acknowledgements

The authors thank their colleagues M. Hübner, M. Jungkurth, A. Kamm and F. Sacher for providing raw data from several archives for analysis and P. E. Carrigan for critically reading the manuscript.

Competing interests statement

The authors declare competing interests: see Web version for details. 